\title{
Fast-modulation imaging with the self-coherent camera
}

\author{
P. Martinez \\ Université Côte d'Azur, Observatoire de la Côte d'Azur, CNRS, Laboratoire Lagrange, France \\ e-mail: patrice.martinez@oca.eu
}

Received 10 August 2019 / Accepted 31 August 2019

\begin{abstract}
Context. Direct detection of exoplanets requires imaging in a highly dynamic range and exquisite image quality and stability. Wavefront error (atmospheric errors, manufacturing errors on optics, cophasing residuals, temperature variations, etc.) will limit the efficiency of this endeavor by creating various flavors of speckles that evolve with different timescales. Active wavefront-error correction using a deformable mirror requires measuring the wavefront aberrations in the science image with high accuracy and in a shorter time than the duration of the dominant speckle lifetime.

Aims. The self-coherent camera (SCC) is a focal plane wavefront sensor exploiting the coherence of light to generate Fizeau fringes in the image plane to spatially encode speckles. The SCC combines a coronagraph and a modified Lyot stop to which a reference channel is added. The conventional SCC is restricted to long-exposure measurements because the light transmitted through the reference channel is limited. The SCC can correct quasi-static aberrations but precludes short-lived atmospheric aberrations from the measurement.

Methods. I propose an alternative to the conventional SCC that I call the fast-modulated SCC. It uses a simplified Lyot stop design and an adequate Fourier filtering algorithm. The theory is established and confirmed by means of numerical simulations.

Results. The SCC theory dictates that the separation between the classical Lyot stop and the reference channel must be larger than 1.5 times the Lyot stop diameter. The fast-modulated SCC allows for the reference channel to be placed anywhere, in particular in the vicinity of the pupil where the maximum of diffracted light is found. This alternative represents a complete game changer for the sensor: full compatibility with any type of coronagraph, easy installation in existing instruments, and versatility by accessing shortand long-time exposure measurements.

Conclusions. While the conventional SCC can almost not be implemented in existing instruments because the optical beam footprint in the instrument must be wide enough to illuminate the reference channel, which is often seen as a significant shortcoming, the fastmodulated SCC does not require any constraint on this. The fast-modulated SCC also relaxes the high sampling requirement to resolve the fringes, which is usually incompatible with the observation of fainter targets because the fringes are larger. The fast-modulated SCC simultaneously counteracts the two original shortcomings of the SCC concept.
\end{abstract}

Key words. instrumentation: high angular resolution - instrumentation: adaptive optics - methods: numerical techniques: high angular resolution - telescopes

\section{Introduction}

Observing sequences have shown that high-contrast imaging is fundamentally limited by speckle noise. Speckle noise comes from wavefront errors caused by various independent sources and evolves on different timescales depending on their nature. Speckles can be divided into three main families: (i) large dynamical wavefront errors generated by the atmosphere; (ii) slowly varying instrumental wavefront aberrations (amplitude and phase errors) of various causes, notably mechanical or thermal deformations resulting in quasi-static speckles; and (iii) long-timescale wavefront errors found in the optical train (e.g., optical quality, misalignment errors, cophasing residuals) resulting in static speckles. Maintaining nearly aberration-free wavefront defects (in both phase and amplitude) over time is essential for any coronagraphic instrument. A high level of wavefront control (correcting for dynamical, quasi-, and static aberrations) and wavefront shaping (creating a dark hole of very deep contrast over a limited area in the field of interest) is paramount. Various wavefront sensors are available and have been proposed over the years for adaptive optics, active optics (telescope and instrument), cophasing optics, etc.
The self-coherent camera (SCC; Baudoz et al. 2006; Galicher et al. 2008) is a wavefront sensor that can be used for both instrument wavefront control and wavefront shaping. The SCC is a powerful tool for exoplanet detection because it works directly on the coronagraphic image. The SCC creates Fizeau interferences to spatially modulate the speckle intensity in the coronagraphic image. From one coronagraphic image it is possible to retrieve the complex electric field, and thus estimate accurately the wavefront errors that are found upstream from the coronagraph (because the SCC is used as a focal plane wavefront sensor downstream from a coronagraph). Many attempts have been made to improve the potential of the sensor for active optics (Galicher et al. 2010; Mazoyer et al. 2013; Mazoyer 2014; Delorme et al. 2016) and fine cophasing optics (Janin-Potiron et al. 2016). The SCC is a low-cost sensor benefiting from elegance and simplicity. However, the main design characteristic of the SCC is also the main drawback of the concept: the additional use of an off-axis pinhole in the Lyot stop, the so-called reference channel. The light transmitted through the reference channel interferes with the main Lyot beam in the downstream focal plane, creating fringes in the detector image. These fringes retain 

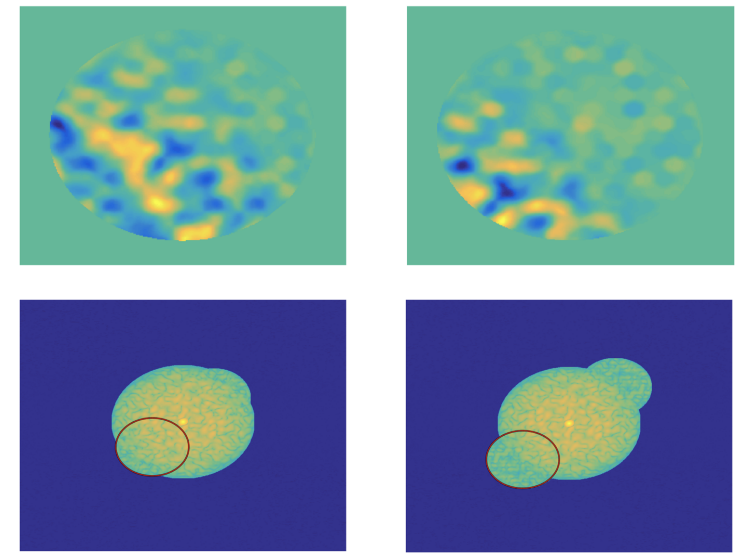
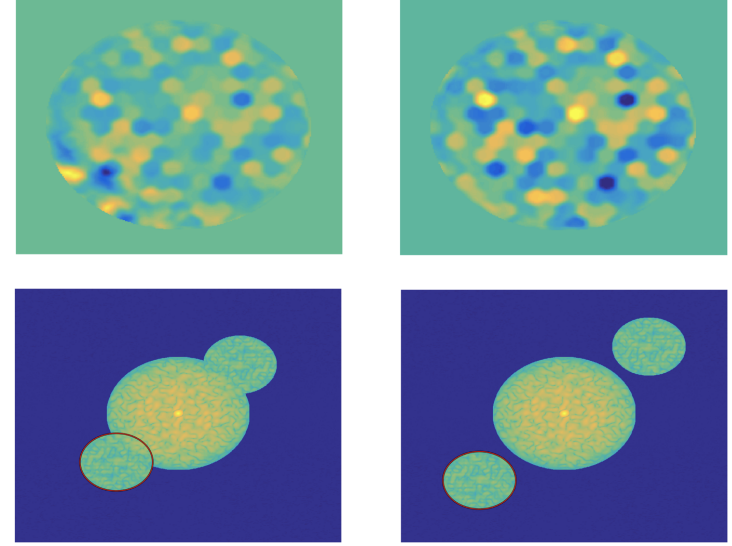

Fig. 1. Effect of the $\beta$ parameter: $0.4,0.6,0.8$, and 1.1 from left to right. Top row: segmented piston phase as estimated by the conventional SCC. Bottom row: corresponding OTFs where the side lobe $I_{-}$is highlighted by the red circle for clarity.

both phase and amplitude information from the complex electric field of a star. Because the amount of light transmitted through the reference channel is limited, present-day SCC only works on long exposures that preclude the correction of fast-living atmospheric aberrations. In contrast with laboratory operation, on-sky operation of the SCC suffers from the reference flux-limitation (Delorme 2016).

A recent study (Gerard et al. 2018) showed that one way to increase the amount of light in the reference channel is to modify the design of the coronagraph and revise the SCC Fourier filtering algorithm. The modification of the SCC is referred to as the fast atmospheric SCC technique (FAST). In this paper, I propose another approach to solve the same issue by revising the Lyot stop design and the SCC Fourier filtering algorithm. The proposed solution is called the fast-modulated SCC and represents a complete game changer because it allows for placing the reference channel anywhere, in particular at the maximum flux position in the Lyot plane. The constrain on the separation between the reference channel and the Lyot stop with the conventional SCC is removed with the fast-modulated SCC.

The paper is structured as follow: in Sect. 2 I briefly recall the principle of the SCC, reference channel properties, and system parameters, and discuss practical limitations. In Sect. 3 I detail the fast-modulated SCC. Section 4 concludes on the gain offered by the fast-modulated SCC strategy.

\section{Self-coherence camera}

\subsection{Principle}

This section briefly recalls the main steps for measuring wavefront errors with the SCC. More details can be found in Baudoz et al. (2006) and Galicher et al. (2008) and subsequent papers. The detailed formalism of the SCC is summarized in Appendix A.

To calibrate speckles, the SCC uses a modified classical Lyot stop by adding a small hole called reference channel that picks up a small amount of stellar light. The science and the reference channels interfere in a Fizeau scheme, and the SCC spatially modulates the stellar speckles on the detector, but it does not affect a potential companion image because its light is not coherent with stellar light.

Pupil plane and focal plane variables are denoted $\epsilon$ and $\alpha$, respectively. The symbol $\lambda$ expresses the observing wavelength. Fourier transform of a function $f$ is noted $\mathcal{F}(f)$, and the inverse Fourier transform is expressed as $\mathcal{F}^{-1}(f)$. The cross denotes the product operator, while the cross in a circle denotes the convolution product. The required pupil separation between the reference and the science channels, to avoid any overlap of the peaks in the spatial frequency plane, Fourier transform of the SCC image $(I)$, the complex-valued optical transfer function (OTF), is denoted $\epsilon_{0}$ (see Fig. 1 bottom right image). With these notations, the SCC interferential image is expressed as

$$
\begin{aligned}
I(\alpha, \lambda)= & \left|A_{\mathrm{S}}\right|^{2}+\left|A_{\mathrm{R}}\right|^{2}+\left|A_{\mathrm{P}}\right|^{2}+A_{\mathrm{S}}^{*} A_{\mathrm{R}} \exp ^{\left(\frac{-2 i \pi \alpha \epsilon_{0}}{\lambda}\right)} \\
& +A_{\mathrm{S}} A_{\mathrm{R}}^{*} \exp ^{\left(\frac{2 i \pi \alpha \epsilon_{0}}{\lambda}\right)},
\end{aligned}
$$

where the first three terms refer to the coronagraphic residuals (stellar, reference, and planet images). The two last terms refer to the modulation components that represent the spatial speckle modulation through interferences with a $\lambda / \epsilon_{0}$ interfringe.

To estimate wavefront errors, the modulated parts of $I(\alpha, \lambda)$ denoted $I_{-}(\boldsymbol{\alpha}, \lambda)$ and $I_{+}(\boldsymbol{\alpha}, \lambda)$ need to be isolated because they contain a linear combination of $A_{\mathrm{S}}$ and $A_{\mathrm{R}}$. Their inverse Fourier transform leads to

$$
\begin{aligned}
\mathcal{F}^{-1}[I](\epsilon, \lambda)= & \mathcal{F}^{-1}\left[I_{\mathrm{S}}+I_{\mathrm{R}}+I_{\mathrm{P}}\right]+\mathcal{F}^{-1}\left[A_{\mathrm{S}}^{*} A_{\mathrm{R}}\right] \otimes \delta\left(\boldsymbol{\epsilon}-\frac{\boldsymbol{\epsilon}_{\mathbf{0}}}{\lambda}\right) \\
& +\mathcal{F}^{-1}\left[A_{\mathrm{S}} A_{\mathrm{R}}^{*}\right] \otimes \delta\left(\boldsymbol{\epsilon}+\frac{\boldsymbol{\epsilon}_{\mathbf{0}}}{\lambda}\right),
\end{aligned}
$$

where $I_{\mathrm{S}}=\left|A_{\mathrm{S}}\right|^{2}, I_{\mathrm{R}}=\left|A_{\mathrm{R}}\right|^{2}$, and $I_{\mathrm{P}}=\left|A_{\mathrm{P}}\right|^{2}$ are intensities of the speckles. The image obtained after this inversion is composed of three entities centered in $\boldsymbol{u}=\left[-\epsilon_{0} / \lambda, 0, \epsilon_{0} / \lambda\right]$. The two lateral peaks are referred to as $\mathcal{F}^{-1}\left[I_{-}\right]$and $\mathcal{F}^{-1}\left[I_{+}\right]$. One of the two lateral peaks is extracted and Fourier transformed. Wavefront errors upstream of the coronagraph can thus be estimated $\left(\phi_{\text {est }}\right)$ from the OTF expression of $I$. It is expressed as

$\phi_{\text {est }}=\mathcal{F}^{-1}\left[\frac{I_{-}}{A_{\mathrm{R}}^{*} M}\right](\epsilon, \lambda)$,

where $M$ is the coronagraphic mask function. The imaginary and real parts of $\phi_{\text {est }}$ give access to the phase and amplitude parts of the wavefront error.

\subsection{Reference properties and limitations}

The diameter of the pupil and the reference channels are denoted $D_{\mathrm{L}}$ and $d_{\mathrm{R}}$, respectively, and $\gamma$ is the ratio parameter such that

$\gamma=\frac{D_{\mathrm{L}}}{d_{\mathrm{R}}}$. 

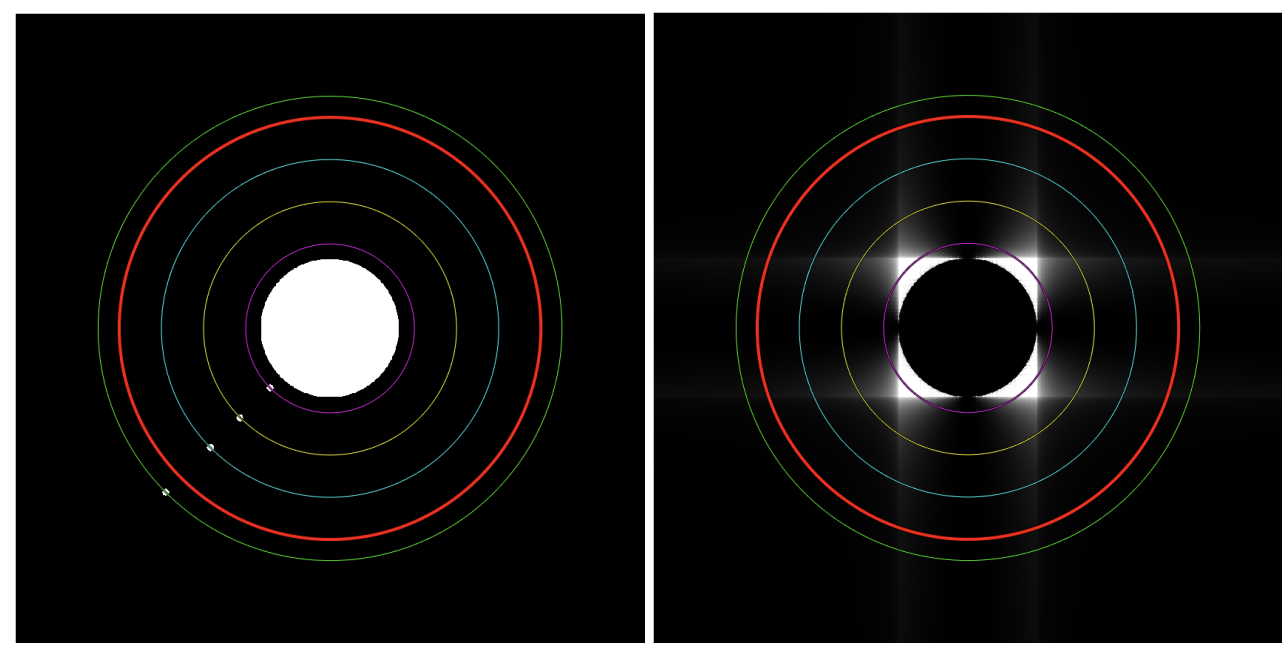

Fig. 2. Illustration of the SCC Lyot stop (left) assuming $\gamma=20$ and diffracted intensity in the Lyot plane with a fourquadrant phase mask coronagraph (right). In both images the area inside the red circle $(\beta=1.0)$ symbolizes the precluded reference positions. The purple, yellow, blue, and green circles correspond to reference positions for which $\beta$ is equal to $0.4,0.6,0.8$, and 1.1 , respectively.

As demonstrated in Galicher et al. (2010), the separation required for $\epsilon_{0}$ to not overlap the side peaks and the central peak is

$\epsilon_{0}=\beta(1.5+0.5 / \gamma) D_{\mathrm{L}}$,

where $\beta$ cannot be lower than unity and is usually set with a $10 \%$ margin $(\beta=1.1)$. If $\beta$ is lower than unity, the phase estimation is compromised and the SCC does not work properly (Fig. 1, the estimated residual increases and is more strongly corrupted with decreasing $\beta$ ). Thus the reference channel has to satisfy a restrictive condition on its position: the center of the pupil and reference channels are at least separated by more than $1.5 \times D_{\mathrm{L}}$ because otherwise the central and lateral peaks would be misidentified (Fig. 1).

Pending the application, the reference must satisfy another condition on its dimension $d_{\mathrm{R}}$ that can be expressed with $\gamma$ as well. I describe this below.

- When the SCC is used as a cophasing sensor (Janin-Potiron et al. 2016), the condition that must be satisfied by $\gamma$ is

$\gamma \geq 4 N_{\text {seg }}+2$,

where $N_{\text {seg }}$ represents the number of segment rings that comprise the telescope pupil. The higher the number of segments or rings $\left(N_{\text {seg }}\right)$, the higher the $\gamma$ value, and the lower the signal in the reference. The higher the value of $\gamma$, the lower the fringe contrast. This translates into typical $\gamma$ values of 10 or 14 for the James Webb Space Telescope (JWST) and the Keck telescope, respectively. For the 798 segments over 16 rings of the Extremely Large Telescope (ELT), the condition transforms into $\gamma \geq 66$, which will undoubtedly lead to a very poor signal-to-noise ratio $(\mathrm{S} / \mathrm{N})$. Expressing the constraint on $d_{\mathrm{R}}$ leads to

$d_{\mathrm{R}} \leq \frac{D_{\mathrm{L}}}{4 N_{\mathrm{seg}}+2}$.

- When the SCC is used for the correction of quasi-static aberrations with a deformable mirror (DM) to guarantee a correction at sufficient $\mathrm{S} / \mathrm{N}$, the maximum reference diameter is (Mazoyer 2014)

$d_{\mathrm{R}} \leq 1.22 \times \sqrt{2} \frac{D_{\mathrm{L}}}{N_{\mathrm{act}}}$,

where $N_{\text {act }}$ is the number of DM actuators across the pupil. This leads to the following condition:

$\gamma \geq \frac{N_{\text {act }}}{1.22 \times \sqrt{2}}$.
Typical values of $\gamma$ would be $29-37$ for $1 \mathrm{~K}$ or $2 \mathrm{~K}$ actuator DM, respectively. The higher the actuator count, the lower the $\gamma$ value, and the lower the signal in the reference. The reference size drives the area in the focal plane that the DM can correct for.

The SCC system includes several fundamental limitations: (i) because the SCC spreads light into fringes, it requires sufficient sampling to detect the fringes that encode the residual speckles; (ii) to optimize the fringe contrast, it is critical to match the intensity distributions and fluxes of pupil and reference channels; (iii) to avoid any overlap of the peaks in the Fourier signal domain, the reference must be distant enough from the pupil channel; and (iv) to guarantee measurement and correction upon application, the size of the reference must be small enough. These limitations prevent a high $\mathrm{S} / \mathrm{N}$ because the coronagraph diffracts light mainly in the vicinity of the pupil channel where the reference channel is not.

\section{Fast-modulated SCC}

\subsection{Breaking the rules}

The SCC theory dictates that the reference cannot be placed in the vicinity of the pupil channel for the reasons mentioned above (in Fig. 2, left, the red circle symbolizes the limit under which the reference cannot be placed). The maximum diffracted energy from the coronagraph can be found in the vicinity of the pupil channel. Figure 2 (right) shows that to obtain the maximum intensity, $\beta \sim 0.4$ is required, while the conventional SCC imposes $\beta>1.0$; the image assumes a four-quadrant phase mask coronagraph (Rouan et al. 2000). The general idea of the fast-modulated SCC is to accommodate the SCC with a reference channel closer to the edge of the pupil channel because it resolves the $\mathrm{S} / \mathrm{N}$ issue of the concept: it relaxes constraints on (ii), suppresses the condition on (iii), and enables the use of the SCC for wider applications in a broader context (iv). It would also relax the requirement on (i) because the fringes are larger when the reference channel is closer (the interfringe is inversely proportional to $\epsilon_{0}$ ).

\subsection{Fast-modulation Fourier filtering algorithm}

The principle of the fast-modulated SCC is simple, and the formalism is detailed in Appendix B. Two SCC images are recorded almost simultaneously using a motorized Lyot stop that closes and opens the reference channel at a high rate. When the reference channel is open, the SCC image is fringed, and it does not 

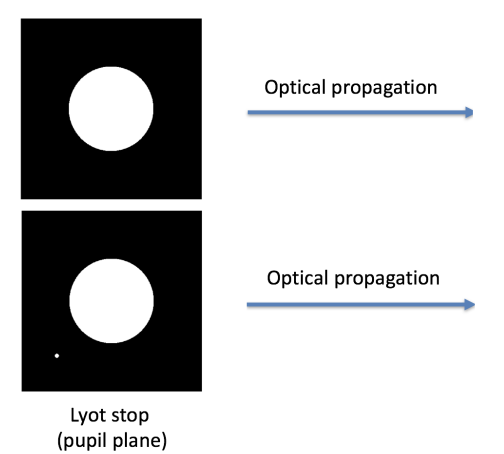

Optical propagation

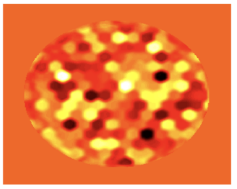

Phase extraction
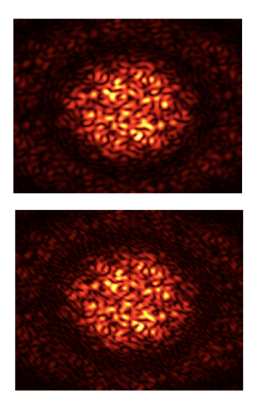

Coronagraphic PSF

(detector plane)

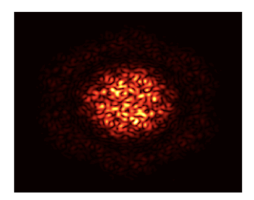

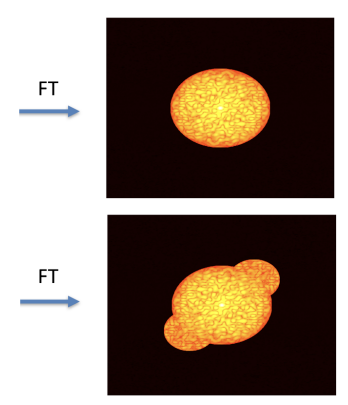

OTF

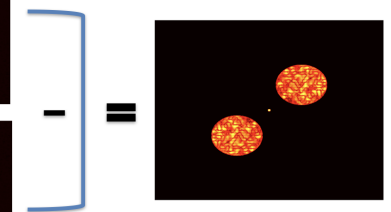

Fig. 3. Fast-modulated SCC Fourier filtering algorithm principle. Two SCC images are obtained (sequentially or simultaneously) with a pupil channel without a reference channel (top, image without fringes) and with a pupil channel with a reference channel (bottom, fringed image) to isolate different SCC image terms in spatial frequency in order to isolate the side lobe of the second OTF.

have a fringe when the channel is closed. The Lyot stop modulator exchanges the two configurations at a high frequency rate dictated by the application, considering integration time and speckle lifetime. This way, the detector can integrate the two component images quasi-simultaneously over various frame exposure times, using the same detection pixels. Both images are subject to exactly the same aberrations and noises. Two OTFs are then computed: one with the three entities, and one with the central entity only. The subtraction of the two allows for recovering the side lobes even for theoretically invalid $\beta$ values (Fig. 3). The rest of the Fourier filtering algorithm is similar to the classical SCC data post-processing. Low $\beta$ value are now possible, for instance, $\beta=0.4$ (Fig. 2 , left), for placing the reference in the vicinity of the pupil channel where a maximum of energy is found. This way, the SCC can integrate the two component images quasi-simultaneously over low frame exposure times. Alternatively, another way to implement the modulated SCC is to consider a differential imaging mode: using two arms where the two Lyot stops (with and without the reference channel) would be installed. Images are recorded simultaneously, but on different detector pixels. In both cases (fastmodulation imaging or differential imaging modes), the Fourier filtering algorithm is the same. Numerical evidence that mirror fine-phasing can be achieved with the fast-modulated SCC is presented in Appendix C.

\subsection{Fast-modulated multireference SCC}

The multireference SCC (MRSCC) has been proposed as an upgrade of the SCC to use the sensor in polychromatic light (Delorme et al. 2016). A unified view of the SCC would consider combining the MRSCC and the fast-modulated SCC principle for simultaneous multitimescale speckle correction. While the fast-modulated SCC suppresses the constraint on the reference position, the multireference SCC would consider placing various references of different sizes, that is, for different applications (atmospheric optics, active optics, cophasing optics, etc.) in the vicinity of the pupil channel. From one set of images with and without fringes the fast-modulated MRSCC would simultaneously work as a cophasing, atmospheric, and dark hole wavefront sensor (assuming that a common exposure integration time can be found). This way, the SCC turns into a complete integrated solution for focal plane wavefront sensing. It paves the way for simultaneous multiple wavefront error measurement or correction regardless of the coronagraph design.

\section{Conclusion}

The conventional SCC offers various major advantages: science image direct measurement with a limited amount of hardware (no dedicated optical path). The fast-modulated SCC retains these advantages and adds one major benefit: while the conventional SCC can almost not be implemented in existing instruments because the optical beam in the instrument must be wide enough to illuminate the reference channel that is installed at a farther distance than three times the pupil radius from the pupil center (which is often seen as a significant shortcoming), the fast-modulated SCC does not place any constraint on this. The fast-modulated SCC only requires a motorized Lyot stop with a modulator on the reference hole. It can therefore be installed in all major present-day high-contrast imaging instruments at almost no cost. Because the reference channel distance to the pupil channel is no longer constrained, the reference channel $\mathrm{S} / \mathrm{N}$ will outperform the conventional SCC S/N. It thus allows for accessing both short- and long-exposure time applications.

\section{References}

Baudoz, P., Boccaletti, A., Baudrand, J., \& Rouan, D. 2006, in IAU Colloq. 200: Direct Imaging of Exoplanets: Science and Techniques, eds. C. Aime, \& F. Vakili, 553

Delorme, J. R. 2016, PhD Thesis, these de doctorat dirigee par Rousset, Gerard et Galicher, Raphael Physique. Astronomie, Astrophysique Sorbonne Paris 7

Delorme, J. R., Galicher, R., Baudoz, P., et al. 2016, A\&A, 588, A136

Galicher, R., Baudoz, P., \& Rousset, G. 2008, A\&A, 488, L9

Galicher, R., Baudoz, P., Rousset, G., Totems, J., \& Mas, M. 2010, A\&A, 509, A31

Gerard, B. L., Marois, C., \& Galicher, R. 2018, AJ, 156, 106

Janin-Potiron, P., Martinez, P., Baudoz, P., \& Carbillet, M. 2016, A\&A, 592, A110

Mazoyer, J. 2014, PhD Thesis, these de doctorat dirigee par Rousset, Gerard et Baudoz, Pierre Astronomie et astrophysique Paris 7

Mazoyer, J., Baudoz, P., Galicher, R., Mas, M., \& Rousset, G. 2013, A\&A, 557, A9

Rouan, D., Riaud, P., Boccaletti, A., Clénet, Y., \& Labeyrie, A. 2000, PASP, 112, 1479 


\section{Appendix A: Formalism of the self-coherent camera}

In the following, I recall the SCC formalism (Baudoz et al. 2006; Galicher et al. 2008; Mazoyer et al. 2013) that leads to the main equations discussed in Sect. 2. It is assumed that the star is onaxis, and no planet signal is considered for the sake of simplicity. Fourier transform of a function $f$ is noted $\mathcal{F}(f)$ and the inverse Fourier transform is expressed as $\mathcal{F}^{-1}(f)$. The cross denotes the product operator, while the cross in a circle denotes the convolution product. The pupil $P$ is defined as the telescope aperture function, $M$ is the coronagraphic focal plane mask function, and $L$ is the Lyot stop function. Pupil plane variables $\left(\epsilon_{x}, \epsilon_{y}\right)$ are described by the vectorial form $(\boldsymbol{\epsilon})$. Similarly, $\boldsymbol{\alpha}$ denotes the focal plane variables. The symbol $\lambda$ expresses the observing wavelength. The quantity $\psi$ denotes the wave complex amplitude. The function $\phi(\epsilon, \lambda)$ represents the phase aberrations that are located in the pupil.

Under these assumptions, the pupil function defined over a diameter $D_{\mathrm{P}}$ is

$$
P(\boldsymbol{\epsilon})= \begin{cases}1 & \text { if }|\boldsymbol{\epsilon}| \leq \frac{D_{\mathrm{P}}}{2} \\ 0 & \text { otherwise. }\end{cases}
$$

Similarly, the Lyot stop function defined over a diameter $D_{\mathrm{L}}$ can be expressed as

$$
L(\boldsymbol{\epsilon})= \begin{cases}1 & \text { if }|\boldsymbol{\epsilon}| \leq \frac{D_{\mathrm{L}}}{2} \\ 0 & \text { otherwise. }\end{cases}
$$

The SCC uses a small reference hole in addition to the classical Lyot stop $(L)$. The separation between the classical Lyot stop and the reference hole is denoted $\epsilon_{0}$ (from center to center), and $\gamma$ expresses the ratio between $D_{\mathrm{L}}$ (the Lyot stop diameter) and the diameter of the reference hole. Under these conditions, the SCC Lyot stop can be expressed as

$L^{\prime}(\boldsymbol{\epsilon})=L(\boldsymbol{\epsilon})+R(\boldsymbol{\epsilon}) \otimes \delta\left(\boldsymbol{\epsilon}-\boldsymbol{\epsilon}_{\mathbf{0}}\right)$,

where

$R(\boldsymbol{\epsilon})= \begin{cases}1 & \text { if }|\boldsymbol{\epsilon}| \leq \frac{D_{\mathrm{L}}}{2 \times \gamma} \\ 0 & \text { otherwise. }\end{cases}$

The complex amplitude field inside the pupil denoted $\psi_{A}(\boldsymbol{\epsilon}, \lambda)$ can be expressed as

$\psi_{A}(\boldsymbol{\epsilon}, \lambda)=P \times \exp (i \phi)$.

Under the Marechal approximation, $\exp (i \phi) \approx 1+i \phi$, leading to

$\psi_{A}(\boldsymbol{\epsilon}, \lambda)=P+i \phi P$

In the first focal plane where the focal plane mask $M$ is inserted, the complex amplitude $\psi_{\mathrm{B}}(\boldsymbol{\alpha}, \lambda)$ is expressed as

$\psi_{\mathrm{B}}(\boldsymbol{\alpha}, \lambda)=\mathcal{F}\left(\psi_{A}(\boldsymbol{\epsilon}, \lambda)\right) \times M(\boldsymbol{\alpha})$,

$\psi_{\mathrm{B}}(\boldsymbol{\alpha}, \lambda)=(\mathcal{F}(P)+i \mathcal{F}(\phi P)) \times M(\alpha)$.

In the Lyot plane where the Lyot mask is inserted, the complex amplitude $\psi_{\mathrm{C}}(\boldsymbol{\alpha}, \lambda)$ is expressed as

$\psi_{\mathrm{C}}(\boldsymbol{\epsilon}, \lambda)=\mathcal{F}^{-1}\left(\psi_{\mathrm{B}}(\boldsymbol{\alpha}, \lambda)\right) \times L^{\prime}(\boldsymbol{\epsilon})$,

$\psi_{\mathrm{C}}(\boldsymbol{\epsilon}, \lambda)=\left((P+i \phi P) \otimes \mathcal{F}^{-1}(M(\boldsymbol{\alpha})) \times\left[L(\boldsymbol{\epsilon})+R(\boldsymbol{\epsilon}) \otimes \delta\left(\boldsymbol{\epsilon}-\boldsymbol{\epsilon}_{\mathbf{0}}\right)\right]\right.$.
In the Lyot plane it is interesting to note that $\psi_{\mathrm{C}}(\boldsymbol{\epsilon}, \lambda)$ can be expressed as the sum of two terms

$$
\begin{aligned}
\psi_{\mathrm{C}}(\boldsymbol{\epsilon}, \lambda)= & \left((P+i \phi P) \otimes \mathcal{F}^{-1}(M(\boldsymbol{\alpha})) \times L(\boldsymbol{\epsilon})\right. \\
& +\left((P+i \phi P) \otimes \mathcal{F}^{-1}(M(\boldsymbol{\alpha})) \times R(\boldsymbol{\epsilon}) \otimes \delta\left(\boldsymbol{\epsilon}-\boldsymbol{\epsilon}_{\mathbf{0}}\right),\right.
\end{aligned}
$$

where the first term is the complex amplitude as in a classical coronagraphic process, and the second term is the contribution added by the reference hole. Finally, in the detector plane the final complex amplitude $\psi_{\mathrm{D}}(\boldsymbol{\alpha}, \lambda)$ is

$\psi_{\mathrm{D}}(\boldsymbol{\alpha}, \lambda)=\mathcal{F}\left(\psi_{\mathrm{C}}(\boldsymbol{\epsilon}, \lambda)\right)$,

which can be written as

$\psi_{\mathrm{D}}(\boldsymbol{\alpha}, \lambda)=[(\mathcal{F}(P)+i \mathcal{F}(\phi P)) \times M(\boldsymbol{\alpha})] \otimes \mathcal{F}\left(L(\boldsymbol{\epsilon})+R(\boldsymbol{\epsilon}) \otimes \delta\left(\boldsymbol{\epsilon}-\boldsymbol{\epsilon}_{\mathbf{0}}\right)\right)$,

and finally can be expressed such as

$$
\begin{aligned}
\psi_{\mathrm{D}}(\boldsymbol{\alpha}, \lambda)= & {[(\mathcal{F}(P)+i \mathcal{F}(\phi P)) \times M(\boldsymbol{\alpha})] \otimes \mathcal{F}(L(\boldsymbol{\epsilon})) } \\
& +[(\mathcal{F}(P)+i \mathcal{F}(\phi P)) \times M(\boldsymbol{\alpha})] \otimes \mathcal{F}(R(\boldsymbol{\epsilon})) \times \mathrm{e}^{-\frac{i 2 \pi}{\lambda} \alpha \epsilon_{0}} .
\end{aligned}
$$

It is interesting to note that the complex amplitude in the detector plane can be separated into two parts,

$\psi_{\mathrm{D}}(\boldsymbol{\alpha}, \lambda)=A_{\mathrm{S}}+A_{\mathrm{R}} \times \mathrm{e}^{-\frac{i 2 \pi}{\lambda} \boldsymbol{\alpha} \epsilon_{0}}$,

where $A_{\mathrm{S}}$ is the complex amplitude as defined in classical coronagraphy $\left(A_{\mathrm{S}}=A^{\mathrm{D}}+A^{\mathrm{A}}\right)$, where

$A^{\mathrm{D}}=(\mathcal{F}(P) \times M(\boldsymbol{\alpha})) \otimes \mathcal{F}(L(\boldsymbol{\epsilon}))$,

(the term is independent of the aberration $\phi$ ), and

$A^{\mathrm{A}}=i(\mathcal{F}(\phi P) \times M(\boldsymbol{\alpha})) \otimes \mathcal{F}(L(\boldsymbol{\epsilon}))$,

which depends on $\phi$. Following these notations,

$A_{\mathrm{R}}=[(\mathcal{F}(P)+i \mathcal{F}(\phi P)) \times M(\boldsymbol{\alpha})] \otimes \mathcal{F}(R(\boldsymbol{\epsilon}))$.

Finally, the intensity of the on-axis star in the detector plane $I(\alpha, \lambda)$ is expressed as the square modulus of $\psi_{\mathrm{D}}(\alpha, \lambda)$

$I(\boldsymbol{\alpha}, \lambda)=\left|A_{\mathrm{S}}+A_{\mathrm{R}} \times \mathrm{e}^{-\frac{i 2 \pi}{\lambda} \alpha \epsilon_{0}}\right|^{2}=\left|A^{\mathrm{D}}+A^{\mathrm{A}}+A_{\mathrm{R}} \times \mathrm{e}^{-\frac{i 2 \pi}{\lambda} \alpha \epsilon_{0}}\right|^{2}$.

The only difference with the intensity as defined in classical coronagraphy is the last term. Because the last term is complex, the square modulus is expressed as the product of the term by its conjugate (the asterisk denotes the conjugate). The intensity is

$I(\alpha, \lambda)=\left|A_{\mathrm{S}}+A_{\mathrm{R}} \times \mathrm{e}^{-\frac{i 2 \pi}{\lambda} \alpha \epsilon_{0}}\right| \times\left|A_{\mathrm{S}}^{*}+A_{\mathrm{R}}^{*} \times \mathrm{e}^{\frac{i 2 \pi}{\lambda} \alpha \epsilon_{0}}\right|$,

and becomes

$I(\alpha, \lambda)=\left|A_{\mathrm{S}}\right|^{2}+\left|A_{\mathrm{R}}\right|^{2}+A_{\mathrm{S}} A_{\mathrm{R}}^{*} \times \mathrm{e}^{\frac{i 2 \pi}{\lambda} \alpha \epsilon_{0}}+A_{\mathrm{S}}^{*} A_{\mathrm{R}} \times \mathrm{e}^{-\frac{i 2 \pi}{\lambda} \alpha \epsilon_{0}}$.

The intensity in the detector plane is composed of two main terms:

$I_{\mathrm{C}}(\alpha, \lambda)=\left|A_{\mathrm{S}}\right|^{2}+\left|A_{\mathrm{R}}\right|^{2}$,

which expresses the sum of the square modulus of the complex amplitude in the Lyot pupil and the reference. Because these quantities are intensities, we cannot access the wavefront error $(\phi)$ term they contain. For the second term left in the intensity expression, the quantities are arbitrarily expressed as $I_{+}=A_{\mathrm{S}} A_{\mathrm{R}}^{*}$ 
and $I_{-}=A_{\mathrm{S}}^{*} A_{\mathrm{R}}$, where it is straightforward to see that $I_{+}$is the conjugate of $I_{-}$, and vice versa. Therefore the intensity in the detector plane can be rewritten as

$I(\boldsymbol{\alpha}, \lambda)=I_{\mathrm{C}}(\boldsymbol{\alpha}, \lambda)+I_{+} \times \mathrm{e}^{\frac{i 2 \pi}{\lambda} \alpha \epsilon_{0}}+I_{-} \times \mathrm{e}^{-\frac{i 2 \pi}{\lambda} \alpha \epsilon_{0}}$,

where the two last terms express the modulation of the speckles and represent the interference term, where the interfringe is defined by the quantity $\lambda / \epsilon_{0}$.

From the fringed coronagraphic image recorded by the detector, the SCC data post-processing starts by numerically computing the inverse Fourier transform (numerical, not optical) of the image, corresponding to the OTF, where

$$
\begin{aligned}
\mathcal{F}^{-1}(I(\boldsymbol{\alpha}, \lambda))=\mathcal{F}^{-1}\left(I_{\mathrm{C}}(\boldsymbol{\alpha}, \lambda)\right) & +\mathcal{F}^{-1}\left(I_{+}\right) \otimes \delta\left(\boldsymbol{\epsilon}-\boldsymbol{\epsilon}_{\mathbf{0}}\right) \\
& +\mathcal{F}^{-1}\left(I_{-}\right) \otimes \delta\left(\boldsymbol{\epsilon}+\boldsymbol{\epsilon}_{\mathbf{0}}\right) .
\end{aligned}
$$

The central entity is expressed in the previous equation by the first term $\mathcal{F}^{-1}\left(I_{\mathrm{C}}(\boldsymbol{\alpha}, \lambda)\right)$ and refers to the sum of the autocorrelation of the electric field in the classical Lyot stop and the reference. The diameter of the central peak is $2 \times \frac{D_{\mathrm{L}}}{\lambda}$. The two lateral peaks correspond to the correlation between the stellar electric fields in the classical Lyot and the reference hole. The two peaks are conjugated, and thus contain the same information. The diameter of these two lateral peaks is $\frac{D_{\mathrm{L}}(1+1 / \gamma)}{\lambda}$.

The term $\mathcal{F}^{-1}\left(I_{-}\right)$is then isolated because it is equivalent to $\mathcal{F}^{-1}\left(A_{\mathrm{S}} A_{\mathrm{R}}^{*}\right)$. Because $\gamma \gg 1$, we can assume $A_{\mathrm{R}}^{*}$ constant such that $A_{\mathrm{R}}^{*}=A_{0}$, and thus $I_{-} \sim A_{\mathrm{S}}$ and $\mathcal{F}^{-1}\left(I_{-}\right) \sim \mathcal{F}^{-1}\left(A_{\mathrm{S}}\right)$.

From $\mathcal{F}^{-1}\left(I_{-}\right)$we can recover $A_{\mathrm{S}}$ using the Fourier transform of $\mathcal{F}^{-1}\left(I_{-}\right)$because $\mathcal{F}\left(\mathcal{F}^{-1}\left(I_{-}\right)\right)=I_{-} \sim A_{\mathrm{S}}$.

We know from Eq. (9) that

$A_{\mathrm{S}}=[(\mathcal{F}(P)+i \mathcal{F}(\phi P)) \times M(\alpha)] \otimes \mathcal{F}(L(\boldsymbol{\epsilon}))$,

from which we wish to extract $\phi$. Multiplying $A_{\mathrm{S}}$ (measured by the SCC) by the inverse of the mask function $\left(\times \frac{1}{M}\right)$ and applying the inverse Fourier transform to the result such that

$\mathcal{F}^{-1}\left(A_{\mathrm{S}} \times \frac{1}{M}\right)=(P+i \phi P) \times L(\boldsymbol{\epsilon})$,

the imaginary and real parts of the result give direct access to the phase and amplitude error, respectively. The estimator $\phi_{\text {est }}$ of the SCC can be written as

$\phi_{\mathrm{est}}=\left[\mathcal{F}^{-1}\left(\frac{I_{-}}{A_{0} M}\right)\right] \times P$.

\section{Appendix B: Principle of the fast-modulated SCC}

The fast-modulated SCC works with two images: a fringed SCC image $\left(I_{1}\right.$, reference channel opened) and an image without fringe $\left(I_{2}\right.$, reference channel closed). The OTF that corresponds to the first image is

$$
\begin{aligned}
\mathcal{F}^{-1}\left(I_{1}(\boldsymbol{\alpha}, \lambda)\right)= & \mathcal{F}^{-1}\left(I_{\mathrm{C}}(\boldsymbol{\alpha}, \lambda)\right)+\mathcal{F}^{-1}\left(I_{+}\right) \otimes \delta\left(\boldsymbol{\epsilon}-\boldsymbol{\epsilon}_{\mathbf{0}}\right) \\
& +\mathcal{F}^{-1}\left(I_{-}\right) \otimes \delta\left(\boldsymbol{\epsilon}+\boldsymbol{\epsilon}_{\mathbf{0}}\right)
\end{aligned}
$$

and the second OTF that corresponds to the second image is

$\mathcal{F}^{-1}\left(I_{2}(\boldsymbol{\alpha}, \lambda)\right)=\mathcal{F}^{-1}\left(I_{\mathrm{C}}(\boldsymbol{\alpha}, \lambda)\right)$.
The subtraction of the two allows recovering the side lobes even for theoretically unauthorized $\epsilon_{0}$ positions for which in $\mathcal{F}^{-1}\left(I_{1}(\alpha, \lambda)\right)$ the lateral terms overlap the central one,

$$
\begin{aligned}
\mathcal{F}^{-1}\left(I_{+}\right) \otimes \delta\left(\boldsymbol{\epsilon}-\boldsymbol{\epsilon}_{\mathbf{0}}\right) & +\mathcal{F}^{-1}\left(I_{-}\right) \otimes \delta\left(\boldsymbol{\epsilon}+\boldsymbol{\epsilon}_{\mathbf{0}}\right)+\Theta\left(I_{1}-I_{2}\right) \\
& =\mathcal{F}^{-1}\left(I_{1}(\boldsymbol{\alpha}, \lambda)\right)-\mathcal{F}^{-1}\left(I_{2}(\boldsymbol{\alpha}, \lambda)\right),
\end{aligned}
$$

where $\Theta\left(I_{1}-I_{2}\right)$ refers to the residual left from a potential imperfect subtraction of the central peak from the two OTFs during the interval of acquisition time $(\Delta t)$ between the two images. This term is likely negligible because the detector integrates both images $\left(I_{1}\right.$ and $\left.I_{2}\right)$ quasi-simultaneously using the same detection pixels, meaning that both images are subject to exactly the same aberrations and noises. It is understood that $\Delta t$ must be shorter than the dominant speckle lifetime that the system aims to correct for. Multiplying the result by a binary mask allows isolating one OTF side lobe, for instance, $\mathcal{F}^{-1}\left(I_{-}\right)$. Afterward, the postprocessing steps are similar to the conventional SCC process in order to obtain $\phi_{\text {est }}$.

\section{Appendix C: Cophasing demonstration}

To demonstrate that the fast-modulated SCC works properly, a cophasing close-loop operation is discussed with numerical simulations. Simulations assume a segmented hexagonal telescope composed of 169 segments over seven hexagonal rings without any central obscuration or secondary mirror supports. Simulations make use of simple Fraunhofer propagators between pupil and image planes, which are implemented as fast Fourier transforms (FFTs) generated with a Matlab code. Matrices are 1024× 1024 pixels, and the focal plane sampling is about 4.5 pixels per $\lambda / D$, where $\mathrm{D}$ is the entrance pupil diameter. No dynamical aberrations are included, and the system is free of aberrations with a spatial frequency that is smaller than the segment size. Monochromatic light of wavelength $\lambda=600 \mathrm{~nm}$ is used, and the coronagraph is a four-quadrant phase mask.

A close-loop performance evaluation was addressed to consider piston errors (Fig. C.1, left) and tip-tilt errors (Fig. C.1, right). The same set of a cophasing error maps $(70 \mathrm{~nm} \mathrm{rms})$ was used in all $\beta$ parameter cases: $0.4,0.6,0.8$, and 1.1 . The latter corresponds to the conventional operation of the SCC, while the three first values are not acceptable by the regular SCC (the SCC diverges at the very first iteration in any cases).

The fast-modulated SCC results demonstrate that the system can work with any $\beta$ value. Convergence is always obtained for piston and tip-tilt errors. Figure C. 2 shows that the wavefront error is invariably measured because the modulation term can be algorithmically isolated in spatial frequency, that is, the side lobes of the OTF are constantly recovered.

It is worth noting that the fast-modulated SCC naturally enhances the sky coverage of the SCC cophasing sensor (SCCPS). This was formerly seen as a significant drawback (JaninPotiron et al. 2016).

Finally, it is important to note that the accuracy of the fastmodulated SCC and regular SCC depend on both the size of the reference channel and the $\mathrm{S} / \mathrm{N}$. In the case of the fast-modulated $\mathrm{SCC}$, decreasing the $\beta$ parameter increases the $\mathrm{S} / \mathrm{N}$ and should translate into a difference in the residual phase that is estimated at each iteration between different $\beta$ configurations. This is not readily observable in Figs. C. 1 and C. 2 because the simulation is noise free and made for illustration purposes only. 
P. Martinez: Fast-modulation imaging with the self-coherent camera
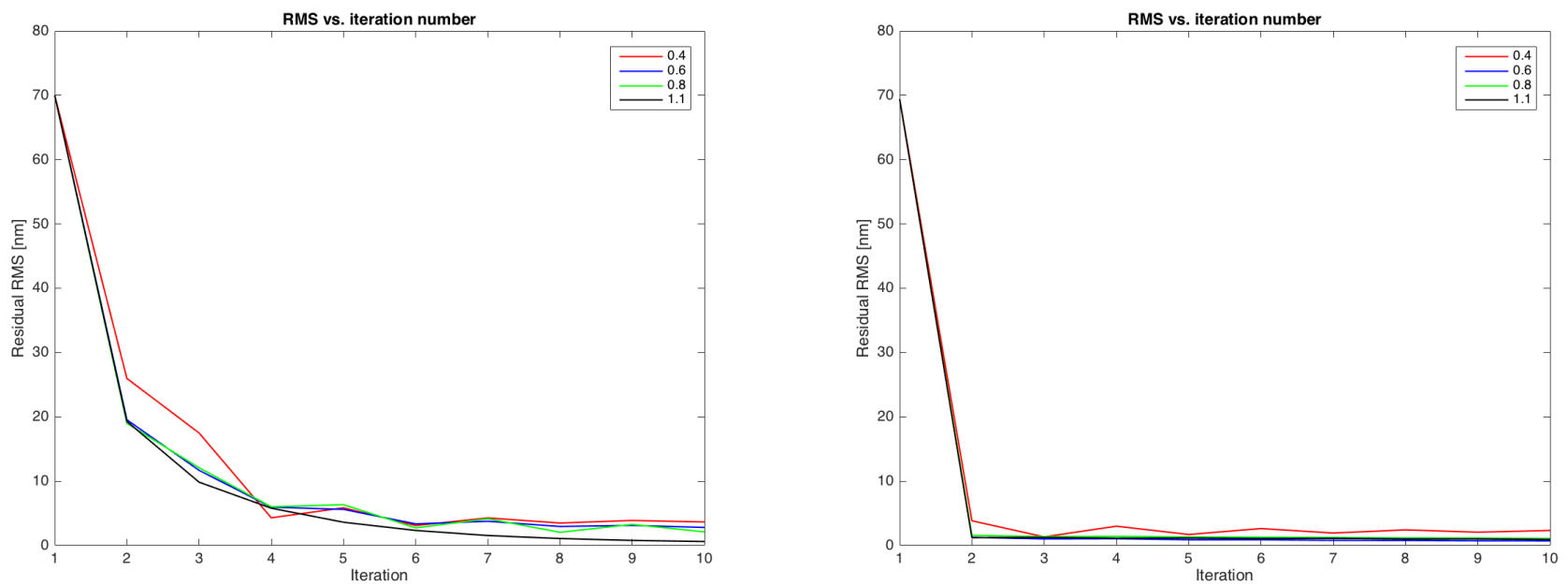

Fig. C.1. rms value evolution of the aberrations over the pupil as function of the iteration number in the case of piston aberrations only (left) and for tip-tilt errors (right). The starting rms value is $70 \mathrm{~nm} \mathrm{rms}$ and the $\beta$ values are given in the captions.
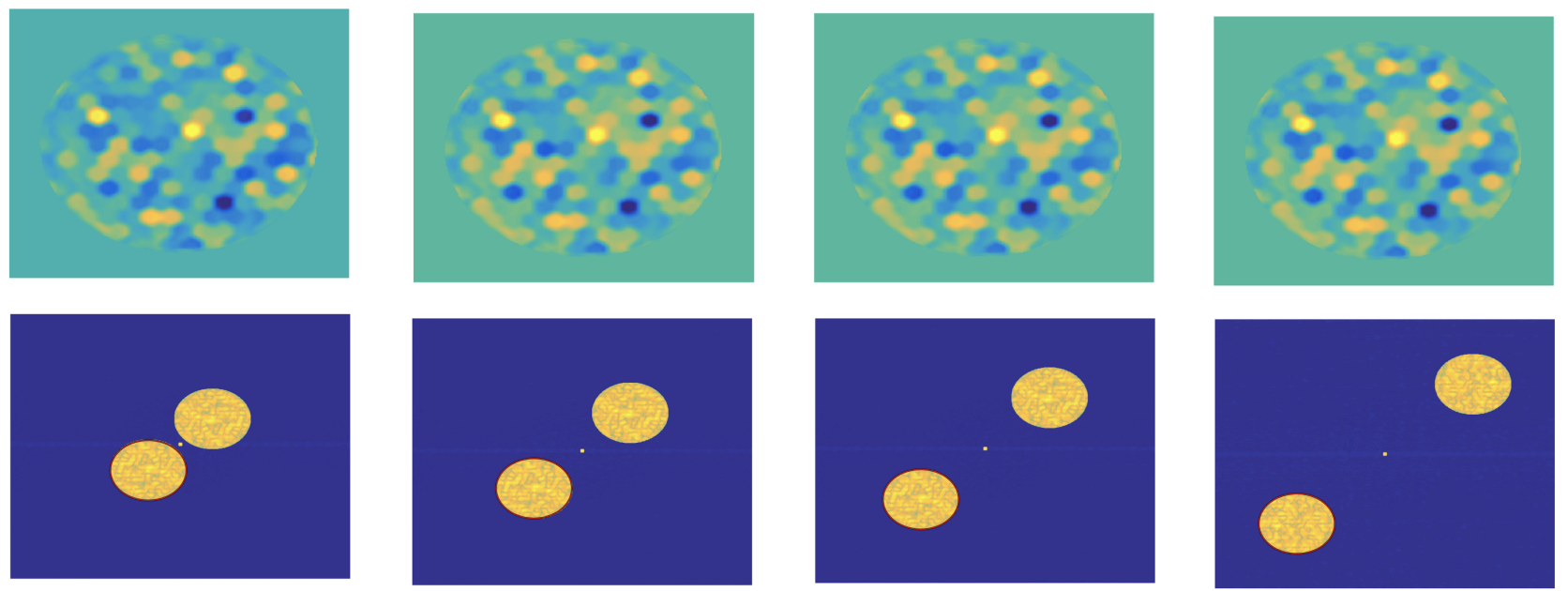

Fig. C.2. Effect of the $\beta$ parameter: 0.4, 0.6, 0.8, and 1.1 from left to right. Top row: segmented piston phase as estimated by the fast-modulated SCC. Bottom row: corresponding OTF where the side lobe $I_{-}$is highlighted by a red circle for clarity. 